\title{
Magnetic Field Change Across the Earth's Bow Shock: Comparison Between Observations and Theory
}

\author{
Daniel Winterhalter, ${ }^{1}$ Margaret G. Kivelson, ${ }^{1}$ Raymond J. Walker, and Christopher T. Russell ${ }^{1}$
}

\author{
Institute of Geophysics and Planetary Physics, University of California, Los Angeles
}

\begin{abstract}
We have examined 204 bow shock crossings observed in the magnetic field and plasma data on ISEE 1. Using the measured upstream field and plasma data, we calculated the downstream field magnitudes by using the single-fluid MHD Rankine-Hugoniot jump conditions, and we compared the results with the observed downstream field magnitudes. We find that the simple MHD model approach can predict the downstream field magnitude only on the average, but does so quite well if (1) a polytropic index of approximately $\frac{5}{3}$ is used in the calculations; (2) the angle between the upstream field and the shock normal is greater than $45^{\circ}$; and (3) the Alfvén Mach (Ma) number is less than about 10 . For angles less than $45^{\circ}$ the predictions overestimate the observed field strength. For $M a>10$ the predictions underestimate the observations.
\end{abstract}

\section{INTRODUCTION}

The properties of a shock wave in a magnetohydrodynamic (MHD) medium such as the solar wind are well approximated by calculations based on the gas dynamic analog of a blunt body in a supersonic flow. Spreiter et al. [1966] showed for the case of the earth's bow shock that their gas dynamic model produces the general geometric characteristics of the bow shock (see also Slavin et al. [1983]) such as the position of the subsolar point, and the shape of the shock particularly near local noon. At the dawn-dusk meridian the geocentric distance to the gas dynamic model shock is about $10 \%$ less than the distance to a surface arrived at by using the best fit to many observed bow shock locations [Holzer et al., 1972] normalized to an average solar wind pressure.

Under certain solar wind conditions the gas dynamic model also estimates the jump in the plasma parameters, such as the density, temperature, and bulk velocity, quite well [e.g., Argo et al., 1967; Spreiter et al., 1968]. Also, under these conditions, the change in the magnetic field vector is predicted well [Spreiter et al., 1966], although the field is not treated in a selfconsistent manner by the gas dynamic theory, i.e., the magnetic field is not allowed to influence the plasma. However, it is clear from the governing equations and the observed range of Alfvén Mach numbers in the solar wind (see Figure $4 a$ and the discussion in section '6) that the interplanetary magnetic field is often important in determining the macroscopic state of the downstream plasma. There are numerous works showing how numerical results for the MHD postshock conditions vary with changing upstream conditions [e.g., Jeffrey and Taniuti, 1964]. Zhuang and Russell [1981] as well, in an analytic examination of the bow shock in an ideal magnetohydrodynamic flow, showed that the plasma parameters immediately behind the shock are influenced by the direction of the solar wind magnetic field. Specifically, they found that for certain field directions the center of isobaric contours shifts away from the subsolar point. In addition, the isodensity contours change shape as a function of interplanetary field direction.

\footnotetext{
'Also at Department of Earth and Space Sciences, University of California, Los Angeles.

Copyright 1985 by the American Geophysical Unıon.

Paper number 4A8215.

$0148-0227 / 85 / 004 A-8215505.00$
}

When the magnetic field effects are important, the gas dynamic description of the bow shock must be modified to include, self-consistently, the solar wind magnetic field. The MHD limit then provides a set of macroscopic conservation equations [de Hoffman and Teller, 1950] generally referred to as the MHD Rankine-Hugoniot equations. With certain simplifying assumptions (see below) these can be solved to obtain the condition of the solar wind plasma downstream of the bow shock purely in terms of the undisturbed upstream solar wind parameters [e.g., Kantrowitz and Petschek, 1966].

Mihalov et al. [1969] were among the first to test the simplest MHD Rankine-Hugoniot jump conditions on the earth's bow shock. Using data from a single Pioneer 6 crossing, they found fair agreement between calculation and measurement, but this agreement was strongly dependent on specific and nonunique methods for determining the shock normal. Because only one bow shock crossing was analyzed, it is not clear whether or not the agreement was fortuitous.

Formisano et al. [1973] briefly addressed the question of the Rankine-Hugoniot equations as applied to the earth's shock with data from several crossings by HEOS 1 . Their results are, however, inconclusive. They agree with the opinion offered by Chao [1972] that the equations probably need to be modified to include effects due to waves and/or turbulence close to the shock surface (see also Chao and Goldstein [1972]).

More recently, Tatrallyay et al. [1984] used more than 300 crossings of the Venus bow shock by the Pioneer Venus orbitor to study how well the MHD and gas dynamic jump conditions apply. They found that the MHD equations approximated the observed jump in the magnetic field at Venus if a polytropic index (ratio of specific heats) of 1.85 for the solar wind was used. Moreover, the index varied with the Alfven Mach number and with the angle between the shock normal and the solar wind magnetic field. They also noted that the MHD solution and the gas dynamic solution became essentially equivalent at and above a plasma beta (ratio of thermal pressure to magnetic pressure) of about 2.7 .

Since its launch in October 1977 the ISEE 1 spacecraft has provided a rather complete and plentiful coverage of bow shock crossings. Using these data, we have conducted a statistical investigation of the applicability of the simple MHD conservation laws to the earth's bow shock, the results of which are presented in this paper.

Mass, momentum, and energy must be conserved across the shock, independent of the specific nature of the interaction. 
The Rankine-Hugoniot equations are an approximate expression of these requirements in the MHD limit and must hold to the degree that the approximations are valid. The purpose of this paper is to compare the Rankine-Hugoniot solutions with observations in order to determine under what solar wind conditions the simple MHD picture of the earth's bow shock applies and under what conditions there is disagreement. Any systematic difference signals the need to account for additional physical processes such as heat flow, turbulence, etc., when setting up the conservation equations. We find that on the average there is good agreement over most of the range in the observed upstream parameters, although there is evidence for systematic differences in some instances. Identification of the mechanisms responsible for these disagreements requires further work.

\section{The Magnetohydrodynamic Jump Conditions}

The magnetohydrodynamic treatment of the effects that the bow shock has on the solar wind is greatly simplified by two factors. First, the typical shock thickness as indicated by the magnetic field ramp is of the order of a few hundreds of kilometers (neglecting for a moment the possibility of upstream wave-particle interactions caused by the shock, and the ion thermalization length downstream). The scale over which the average plasma parameters in the solar wind change, however, is of the order of a few earth radii $\left(R_{E}\right)$. Thus the shock may be considered as a thin discontinuity. Second, the equations describing the conservation of mass, momentum, and energy, and Maxwell's equations, may be integrated across the shock to relate explicitly the fluid properties of one region to those of the other region, without regard to the structure of the shock.

Assuming time independence, the conservation equations may be written [e.g., Boyd and Sanderson, 1969]

$$
\begin{aligned}
\nabla \cdot(\rho \mathbf{U}) & =0 \\
\nabla \cdot(\mathbf{I}) & =0 \\
\nabla \cdot(\mathbf{S}) & =0
\end{aligned}
$$

where $\rho$ is the plasma density, $\mathbf{U}$ is the bulk velocity, $\boldsymbol{\Pi}$ is the total momentum flux tensor, and $\mathbf{S}$ is the total energy flux vector. We define the last two quantities as

$$
\Pi_{\imath j}=\rho U_{1} U_{j}+P_{i j}+\Gamma_{i j}
$$

where $\mathbf{P}$ is the general pressure tensor and $\boldsymbol{\Gamma}$ is the Maxwell stress tensor, and

$$
\mathbf{S}=\left[\frac{3}{2} n k T+\frac{1}{2} \rho U^{2}\right] \mathbf{U}+\mathbf{P} \cdot \mathbf{U}+(\mathbf{c} / 4 \pi)(\mathbf{E} \times \mathbf{B})+\mathbf{Q}
$$

where $\mathbf{Q}$ is the heat flux vector, $T$ is the temperature of the plasma, and $n$ is the number density. The approximation was made here that the solar wind may be described as a singlecomponent fluid obeying charge neutrality and having a single temperature, i.e., the density and temperature are

$$
\begin{gathered}
\rho=n_{+} m_{+}+n_{-} m_{-}=n m_{+}\left(1+m_{-} / m_{+}\right) \sim n m_{+} \\
T=T_{+}+T_{-}
\end{gathered}
$$

where the plus and minus stand for the ion and electron species.

To solve the conservation equations (1) to (3), we now simplify the pressure tensor by assuming hydrostatic pressure (i.e., no viscosity) both upstream and downstream. The heat flux vector $\mathbf{Q}$ in (5) is neglected in our calculations as well. The Maxwell stress tensor in (4) is simplified by taking $E \ll B$, and the Poynting flux in (5) is reexpressed by assuming frozen-in flux (i.e.; no resistivity). Also, using the perfect gas law, the first law of thermodynamics, and the ratio of specific heats $(\gamma)$, one may replace the $\frac{3}{2} n k T$ term in (3) with $\rho I$, where the internal energy $I=P /(\gamma-1) \rho$.

The integration of the conservation equations (1) to (3) is done, in practice, by assuming a planar shock surface which has a normal $\hat{\mathbf{N}}$. All the quantities are relative to a reference frame in which the shock is at rest. In addition, one may choose the fluid velocity to be along the normal, and let $\mathbf{U}_{1}$, $\mathbf{B}_{1}$, and $\hat{\mathbf{N}}$ be coplanar by transforming to a frame moving appropriately along the shock surface (the subscripts 1 and 2 refer to the regions upstream and downstream of the bow shock, respectively). This reduces the divergences in (1), (2), and (3) to one-dimensional derivatives, rendering the integrations trivial. The planar shock approximation is a good one since the curvature of the earth's bow shock is very small, of the order of $10^{-5} \mathrm{~km}^{-1}$ at the nose, and decreasing toward the flanks.

With these definitions and approximations one obtains the jump conditions, or Rankine-Hugoniot equations, representing the conservation of mass, momentum, and energy for the flow of a magnetized fluid through the shock:

$$
\begin{gathered}
{[\rho \mathbf{U} \cdot \hat{\mathbf{N}}]=0} \\
{\left[\mathbf{U} \cdot \hat{\mathbf{N}}\left\{\left(\rho I+\rho U^{2} / 2+B^{2} / 8 \pi\right)+\left(P+B^{2} / 8 \pi\right)\right\}\right.} \\
-(\mathbf{B} \cdot \hat{\mathbf{N}})(\mathbf{B} \cdot \mathbf{U}) / 4 \pi)]=0 \\
{[\mathbf{B} \cdot \hat{\mathbf{N}}]=0 \quad[\hat{\mathbf{N}} \times(\mathbf{U} \times \mathbf{B})]=0}
\end{gathered}
$$

where the last two equations give the jump of the magnetic field (see, for example, Boyd and Sanderson [1969]). The square brackets are the usual notation for the difference between the two sides of the discontinuity. This set of equations may be solved to find all the downstream fluid parameters in terms of the upstream solar wind conditions [e.g., Jeffrey and Taniuti, 1964; Tidman and Krall, 1971].

\section{Data}

We selected 204 bow shock crossings from ISEE 1 magnetic field and plasma data covering the period from 1977 to 1979 . Figure 1 is an ISEE 1 magnetometer plot [Russell, 1978] of a typical crossing which occurred on December 25, 1978. We will use this event as an example to show how the upstream and downstream parameters were selected for this study. The plot of the magnetic field uses 12-s running averages centered on 4-s intervals. The data are in spacecraft coordinates which are approximately the same as geocentric solar ecliptic coordinates (GSE). From the field magnitude we see that the spacecraft was in the solar wind until about 1555 UT, when it encountered the bow shock and entered the magnetosheath. The field strength increased by a factor of about 2 at the shock.

In order to determine the change in the field magnitude across the shock we first took 5-min averages of the components both upstream and downstream from the shock ramp, and then calculated the field magnitudes from these averages. It is very important, especially in the presence of waves or turbulence, that we calculate the magnitude by using the averaged components. If an average of the magnitude is used, the results will be quite different. This point is discussed in more detail in section 6 . 


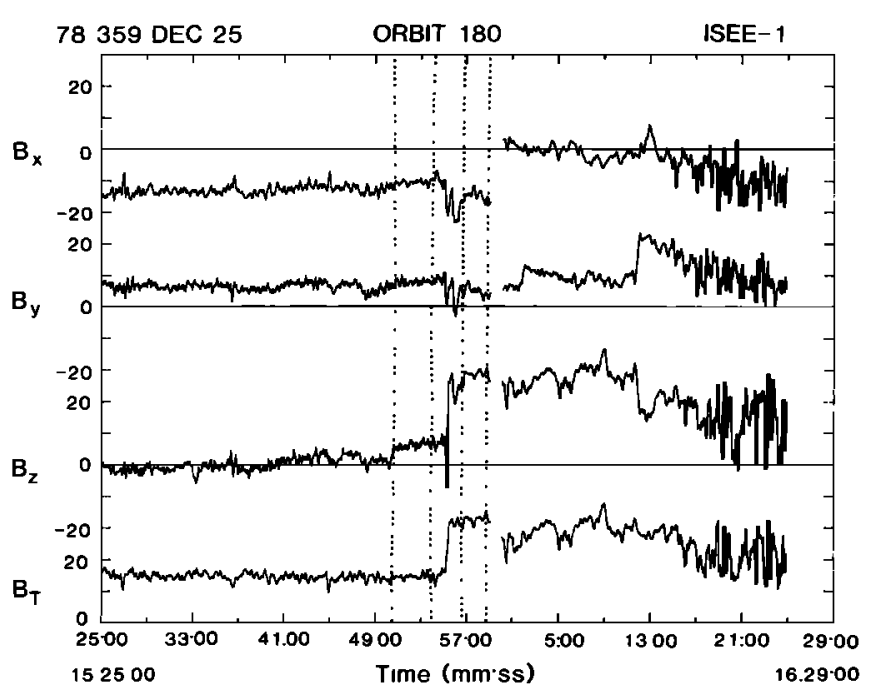

Fig. 1. Representative bow shock crossing as seen in the magnetic field data. The dotted vertical lines are the averaging intervals for the upstream and downstream measurements.

In order to be sure that the measurements were taken well clear of the actual interaction region, the 5-min averaging intervals in front of and behind the shock were chosen to lie upstream of the foot and downstream of the undershoot, when those features were present. In general, we tried to stay clear of any structure associated directly with the shock ramp. If a discontinuity not associated with the bow shock intruded into a measuring interval, the interval was shortened toward the shock ramp in order to exclude the discontinuity. The representative crossing shown in Figure 1 illustrates the last point. Here we see a small discontinuity upstream at about 1550 and a larger one downstream at about 1559. Both were excluded from the measuring intervals depicted by the two pairs of vertical lines.

In Figures $2 a, 2 b$, and $2 c$ the spacecraft positions during the 204 bow shock crossings are displayed in the ecliptic plane, the noon-midnight meridian plane, and the dawn-dusk meridian plane, respectively. There is good local time coverage from dawn to near dusk. Also, all the crossings took place between zero and $10 R_{E}$ above the ecliptic plane.

Figure $3 a$ shows how the upstream magnetic field strength was distributed for the 204 cases, and Figure $3 b$ shows the angle between the upstream field vector and the model shock normal, $\theta_{B N}$. Since in our study the sign of the field vector is immaterial, we chose it such that $\theta_{B N} \leq 90^{\circ}$. The shock normal is discussed in more detail in section 4 . While most of the events (140) have $\theta_{B N}>45^{\circ}$, a significant fraction of them $(33 \%)$ lie in the quasi-parallel regime $\left(\theta_{B N}>45^{\circ}\right)$.

The upstream plasma data were measured by the ISEE 1 solar wind plasma analyzer [Bame et al., 1978]. The data were provided to us in tabular form containing 5-min averages of the solar wind bulk velocity vector, the number density, and the ion temperature. From these tables we selected the 5-min averages corresponding most closely to the time intervals established by the upstream magnetic field averaging procedure described above. For the electron temperature we used a constant value of $1.3 \times 10^{5} \mathrm{~K}$. This value is the most probable electron temperature at one AU [e.g., Feldman et al., 1977]. Also, because of the large heat conductivity of the electrons, their temperature is known to be much less variable than that of the ions [e.g., Hundhausen, 1972].
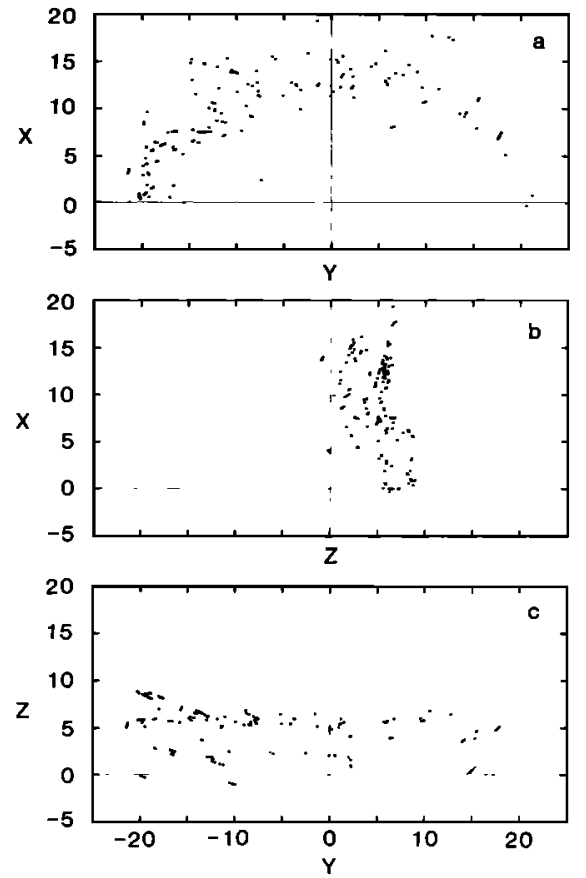

Fig. 2. Observed bow shock crossings (spacecraft coordinates) in (a) the equatorial plane, $(b)$ the noon-midnight meridian plane, and (c) the dawn-dusk meridian plane.

Figure 4 shows the Allvén and sonic Mach numbers and the plasma beta of the upstream solar wind for the 204 cases. The Mach numbers were calculated by using the velocity component normal to the bow shock. The Alfven Mach number has a most probable value between 6 and 7 , but for a large fraction $(45.1 \%)$ of the solar wind observations, values fell between 2 and 6 (Figure $4 a$ ). Also of interest is the fact that nearly $10 \%$ of the cases show the solar wind to have an Alfvén Mach number greater than 10, with the largest being 28 (not shown in Figure $4 a$ [Winterhalter et al., 1984]). The most
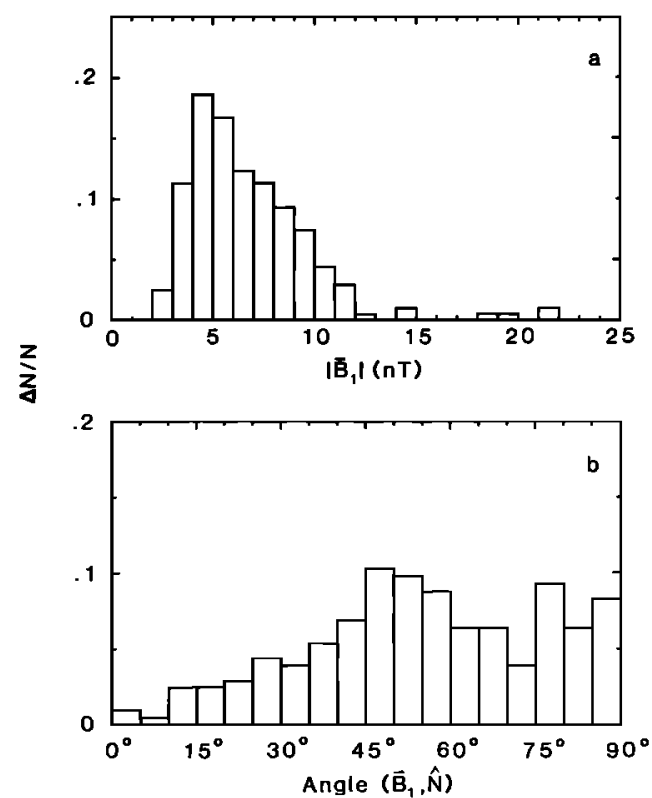

Fig. 3. (a) Distribution of the interplanetary magnetic field strength in our data set. (b) Distribution of the angle between the field vector and the model normal. 

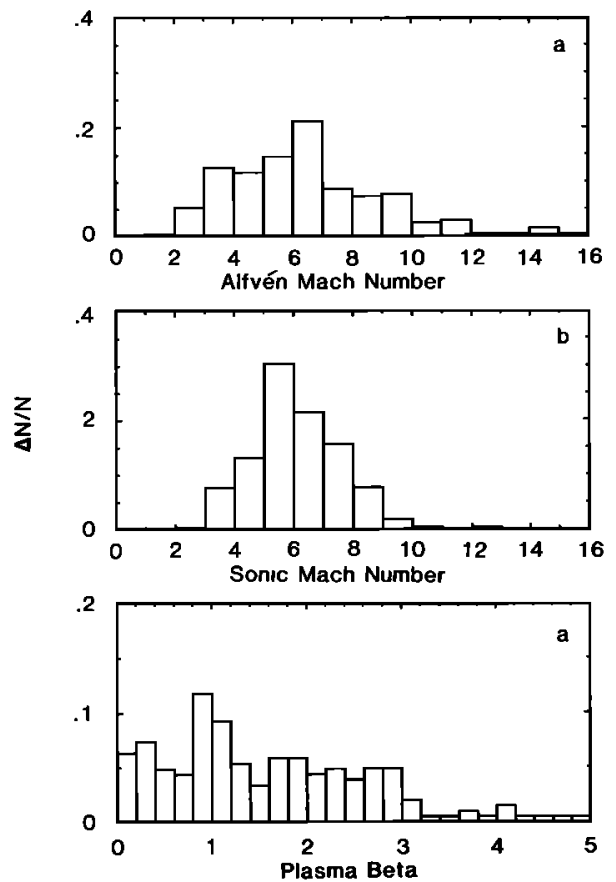

Fig. 4. Distributions of (a) the Alfvén Mach number, (b) the sonic Mach number, and $(c)$ the plasma beta, from the 204 solar wind measurements. The Mach numbers were calculated using the velocity component normal to the bow shock.

probable sonic Mach numbers were between 5 and 8 (Figure $4 b)$.

The distribution of the plasma beta is fairly flat in the range from zero to about 3 , with a small increase for values just less than 1 (Figure $4 c$ ).

\section{The Bow Shock Normal}

Because the jumps in the flow parameters and, especially, in the magnetic field depend sensitively on the geometry of the interaction, an important input parameter to the RankineHugoniot calculations is the bow shock normal vector. Typically, the normal is obtained either from the magnetic coplanarity method or from a model in which a conic section represents the average shape and position of the bow shock surface. The coplanarity theorem [e.g., Colburn and Sonett, 1966; Sonnerup and Cahill, 1967] asserts that the plane containing the upstream and the downstream magnetic field vectors must be perpendicular to the shock plane. If we use this theorem and require the field to be divergence-free, then the normal to the shock surface is given by

$\widehat{\mathbf{N}}= \pm\left(\mathbf{B}_{2}-\mathbf{B}_{1}\right) \times\left(\mathbf{B}_{2} \times \mathbf{B}_{1}\right) /\left(\mathbf{B}_{2}-\mathbf{B}_{1}\right) \times\left(\mathbf{B}_{2} \times \mathbf{B}_{1}\right) \mid$

where $B_{1}$ and $B_{2}$ are the fields upstream and downstream, respectively.

One of the simplest modeling procedures for the bow shock was originated by Holzer et al. [1972], who used an earthcentered conic section with azimuthal symmetry about the earth-sun line. Such a curve is described by

$$
r=L /(1+\varepsilon \cos \alpha)
$$

where $r$ is the radial distance to the shock, $L$ is the semi-latus rectum (the radial distance to the shock in the dawn-dusk plane), $\alpha$ is the sun-earth-satellite angle, and $\varepsilon$ is the eccentricity. Using OGO 1 observations of the shock and applying a least squares fit to these locations, they obtained $\varepsilon=0.7$ and
$L=23.5 R_{E}$. Other models exist, and a good comparison of them is given by Slavin and Holzer [1981].

Applying these two methods to our 204 bow shock crossings, we find that the angle between the two normals varies from zero to $136^{\circ}$. The large deviations of the coplanarity normals from the model normals do not seem reasonable. One explanation for the frequent large angles between the coplanarity and model normal is that they may represent errors caused by the time delay between upstream and downstream field measurements. To check on this possibility, we looked for, and found, a very few (eight) crossings where ISEE 1 and ISEE 2 straddled the bow shock, i.e., while the first spacecraft was in the solar wind, the second already had entered the magnetosheath. The separation between the two spacecraft was typically $1000 \mathrm{~km}$. This provided us with the opportunity to measure the upstream and downstream fields simultaneously, an ideal test of the coplanarity theorem. We found that the two-spacecraft coplanarity normals as well varied through a large range of angles with respect to the model normal, in a manner similar to the one-spacecraft normals. Even though there are very few points, this result suggests that it is not the time delay exclusively that causes the single-spacecraft coplanarity normals to be distributed through a large range of angles relative to the model normals. However, we also note that the coplanarity normals obtained from the eight twospacecraft measurements differed significantly from those obtained from the single-spacecraft measurements. These differences may be partially attributed to the time delay which causes the single-spacecraft normal to be in error. Also, there are errors inherent in applying the coplanarity method, and this point we discuss next.

In Figure 5 we plot the angle between model and coplanarity normal, $\delta$, versus $\theta_{B N}$. The large solid circles with the error bars are the $\left(5^{\circ}\right)$ bin averages. We see that for $\theta_{B N}<75^{\circ}$, $\delta$ has a mean value of $25^{\circ} \pm 2^{\circ}$. At $\theta_{B N}=75^{\circ}$ the mean increases suddenly to $57^{\circ} \pm 5^{\circ}$.

In order to understand this, we asked what average angle

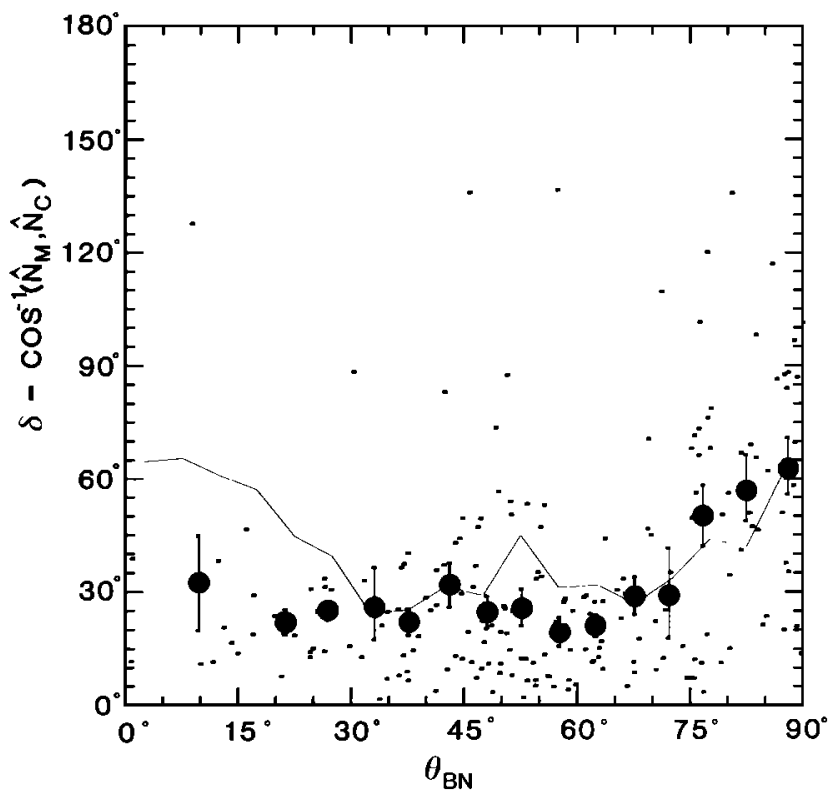

Fig. 5. Angle between model normal and coplanarity normal, $\delta$, versus $\theta_{B N}$. The average of the mean of the angle, $\langle\delta\rangle$, is $25^{\circ} \pm 2^{\circ}$ for $\theta_{B N}<75^{\circ}$. For $\theta_{B N}>75^{\circ},\langle\delta\rangle=57^{\circ} \pm 5^{\circ}$. The solid curve represents the result of a Monte Carlo simulation, calculating $\delta$ with random noise superimposed on arbitrary field components. 
between a constant normal and a normal calculated with (11) might be obtained, recognizing that the upstream and downstream field measurements have a certain amount of random error associated with them. Because the normal direction (equation (11)) is indeterminate when $B_{1}$ and $B_{2}$ are colinear $\left(\theta_{B N}=0^{\circ}\right.$, and $\left.\theta_{B N}=90^{\circ}\right)$, small random errors can produce large uncertainties in the calculated normals.

We simulated the situation with a Monte Carlo approach (suggested by J. Scudder, personal communication, 1984), by superimposing random noise on arbitrary upstream and calculated downstream field components. The upstream field components were varied to let $\theta_{B N}$ range from $0^{\circ}$ to $90^{\circ}$ in steps of $5^{\circ}$. For each upstream and downstream field component the random noise was taken from a Gaussian distribution centered on the $5^{\circ}$ bin mean of the respective observed component, and wih a standard deviation equal to the binaveraged standard deviation of the observed component. The result of this simulation is shown in Figure 5 as the solid curve. We see that above $30^{\circ}$ the difference between coplanarity and model normal is well explained by the noise in the upstream and downstream field measurements. Below $30^{\circ}$ the coplanarity calculations seem to do better than predicted, but this may have resulted from the smaller number of points there. At any rate, we note that typical solar wind magnetic field noise levels cause errors in the coplanarity normal calculations, which are on the average about $25^{\circ}$ for $30^{\circ}<\theta_{B N}<$ $75^{\circ}$, and up to about $60^{\circ}$ for the quasi-perpendicular and quasi-parallel cases.

It may also be that the local surface orientation of the shock differs significantly at times from the average orientation, represented by the model normal. In our Rankine-Hugoniot calculations, however, we are most interested in the average conditions of the solar wind and the boundary, not the small structures, so that the model normal may be the relevant parameter. In addition, the scatter of our results was substantially decreased by the use of the model normal as opposed to the coplanarity normal. For example, the mean of the quantity

$$
\left|B_{2 \mathrm{MHD}}-B_{2 \mathrm{obs}}\right| /\left(B_{2 \mathrm{MHD}}+B_{2 \mathrm{obs}}\right)
$$

was reduced by $67 \%$ when we used the model normal for calculating $\boldsymbol{B}_{2 \mathrm{MHD}}$. For these reasons we will use the normal calculated by using the model [Holzer et al., 1972] in all our calculations.

\section{ANAlysis}

For each of the 204 crossings we used the upstream solar wind parameters, described in section 3 , as input and calculated the downstream magnetic field magnitude with a computer code which is based on the MHD jump conditions of section 2. The calculated downstream magnetic field magnitude was then compared to the actually measured downstream field magnitude in each case.

Figure 6 shows the result of the comparison as a plot of the calculated jump in the field strength $B_{2 \mathrm{MHD}} / B_{1}$ versus the observed jump in the field strength $B_{2 \text { obs }} / B_{1}$ for three different ratios of specific heats (polytropic index), $1.40, \frac{5}{3}$, and 1.85 . The upstream field strength $B_{1}$ is always a measured quantity. Points for which there is agreement between calculation and observation are those on the diagonal line; it is obvious that a large amount of scatter is present in all three panels.

To bring out any trend in the data, we grouped the points into bins of size $B_{2 \mathrm{MHD}} / B_{1}=0.5$. The circles with the error bars are the bin averages. The error bars here and in all subsequent figures represent the error of the mean (i.e., error
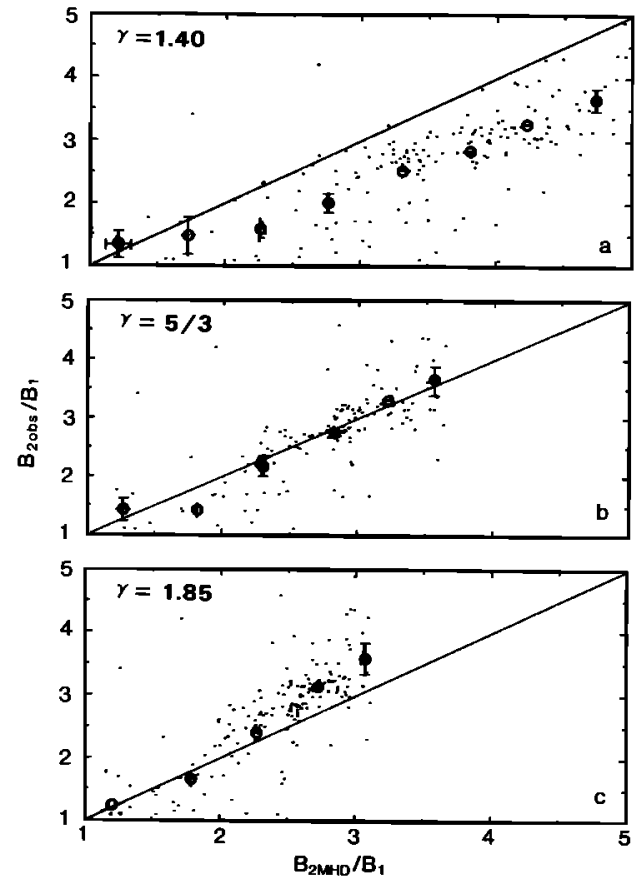

Fig. 6. Comparison between observed jumps and calculated jumps of the field magnitude across the shock, for $\gamma=(a) 1.40,(b) \frac{5}{3}$, and $(c)$ 1.85. The upstream field magnitude $B_{1}$ is always a measured quantity. The error bars on the bin averages (circles) indicate the error of the means. The bin sizes are 0.5 .

$(\bar{z})= \pm \sigma_{\bar{z}} / N^{1 / 2}$, where $\sigma_{\bar{z}}$ is the standard deviation of the bin mean, $\bar{z}$, and $N$ is the number of points in the bin). If no error bars are shown, it means that they are smaller than the symbol used to indicate the average. When calculating the mean in Figure 6, we excluded any points that were more than three standard deviations away from the mean in each bin, so as not to put too much weight on a very few extreme points. This means we excluded less than $3 \%$ of the total population, and no more than $8 \%$ in any one bin.

In the middle panel, for $\gamma=\frac{5}{3}$ (Figure $6 b$ ), the predictions match the observations well on the average. Tatrallyay et al. [1984] found that the best fit to their data for the Venus bow shock was obtained from calculations using the polytropic index $\gamma=1.85$. The bottom panel, Figure $6 c$, shows clearly, however, that this is not the case for the earth's bow shock. A polytropic index of 1.85 underestimates the magnetic field jump in this data set, at least for observed jumps greater than 2. We also included, for contrast, the results obtained when $\gamma=1.40$ (Figure 6a). In this case the slope of the averages is too small to match the observed jumps.

In order to obtain the distribution of the polytropic index for the events analyzed, we let $\gamma$ be a free parameter and varied it until the calculated downstream field value equaled the observed downstream field value, for each of the 204 bow shock crossings. Figure 7 is a histogram of $\gamma$ thus obtained. The distribution ranges from 1 to 2.5 or 3 , with some points scattered even higher, and it has a prominent peak indicating a most probable value between 1.6 and 1.7.

While we expect that the polytropic index of the solar wind is near $\frac{5}{3}$ (see section 6), the considerable width of the distribution in Figure 7 needs to be explored further. In particular, it must be established if the spread solely represents random errors in the input measurements, or if part of it is introduced systematically by upstream solar wind conditions not taken into account by our calculations. To investigate this possi- 


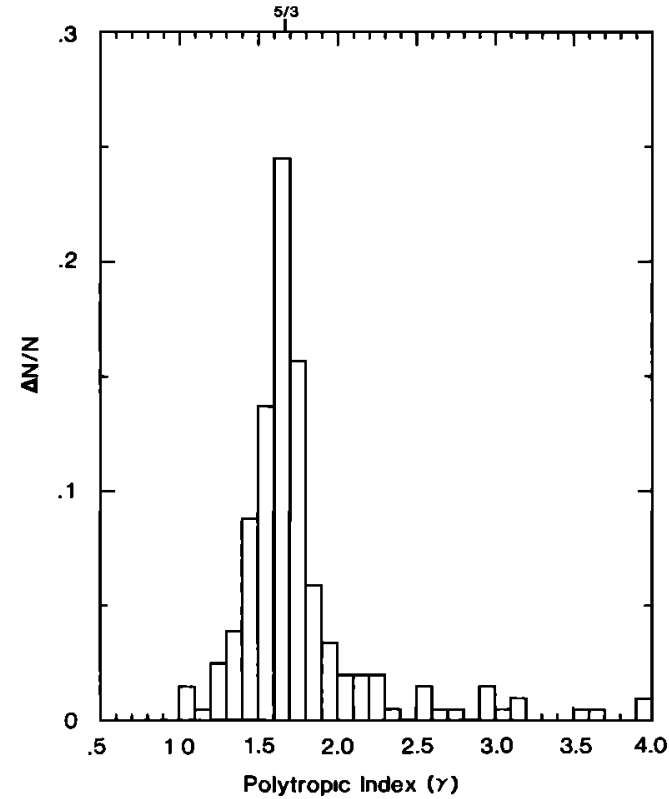

Fig. 7. Distribution of the polytropic index $\gamma$ of the solar wind as inferred from our data sel by requiring $B_{2 \text { MHD }}=B_{2 \text { obs. }}$

bility, we plotted the ratio of calculated to observed downstream field magnitudes $B_{2 \text { MHD }} / B_{2 \text { obs }}$ versus three pertinent solar wind parameters: the angle between the upstream field vector and the bow shock normal, $\theta_{B N}$; the Alfvén Mach number $M a$; and the plasma beta $\beta$, in Figures 8, 9, and 10, respectively.

In Figure 8 the points were grouped into $5^{\circ}$ bins and averaged, again excluding points farther away than three standard deviations from the bin average. The averages indicate good agreement between calculations and observations for angles from $90^{\circ}$ to about $50^{\circ}$ (the quasi-perpendicular regime). In the bins from $35^{\circ}$ to $50^{\circ}$, however, the calculated field values markedly overestimate the observed ones, with a possible peak in the $40^{\circ}$ to $45^{\circ}$ bin. The scatter of the individual points is quite small in the quasi-perpendicular regime. At $45^{\circ}$ to $50^{\circ}$, however, the scatter switches on rather suddenly and stays at high levels throughout the quasi-parallel region ( $\leq$ $\left.45^{\circ}\right)$.

In Figures $9 a-9 c$ we plot the ratio $B_{2 \mathrm{MHD}} / \boldsymbol{B}_{2 \mathrm{obs}}$ versus the Alfvén Mach number. The averages are calculated in unit Mach number bins, excluding points more than two standard deviations away from the mean. The averages in Figure $9 a$ show some disagreement between $B_{2 \mathrm{MHD}}$ and $\boldsymbol{B}_{2 \mathrm{obs}}$. Separating the points for $\theta_{B N}>45^{\circ}$ (Figure $9 b$ ) and $\theta_{B N} \leq 45^{\circ}$ (Figure 9c), we find that the disagreement occurs mainly in the quasiparallel regime. Figure $9 b$ indicates that $B_{2 \mathrm{MHD}} / \boldsymbol{B}_{2 \mathrm{obs}}$ is independent of $M a$ from 2 up to about 10, within error bars, for the quasi-perpendicular crossings. Above $M a=10$ the ratio falls below 1 ; i.e., the calculations underestimate the field jumps. For these Mach number values, however, the number of points is relatively small, and caution must be used when interpreting this result. Figure $9 c$ shows the large amount of scatter present in the quasi-parallel results. There, $\boldsymbol{B}_{2 \mathrm{MHD}} / \boldsymbol{B}_{2 \mathrm{obs}}$ is in general greater than 1.

Figure $10 a$ shows a plot of $B_{2 \mathrm{MHD}} / \boldsymbol{B}_{2 \mathrm{obs}}$ versus the solar wind plasma beta. The points are grouped into bins of size $\beta=0.25$ and averaged, neglecting points which are farther than two standard deviations away. We see that the ratio is

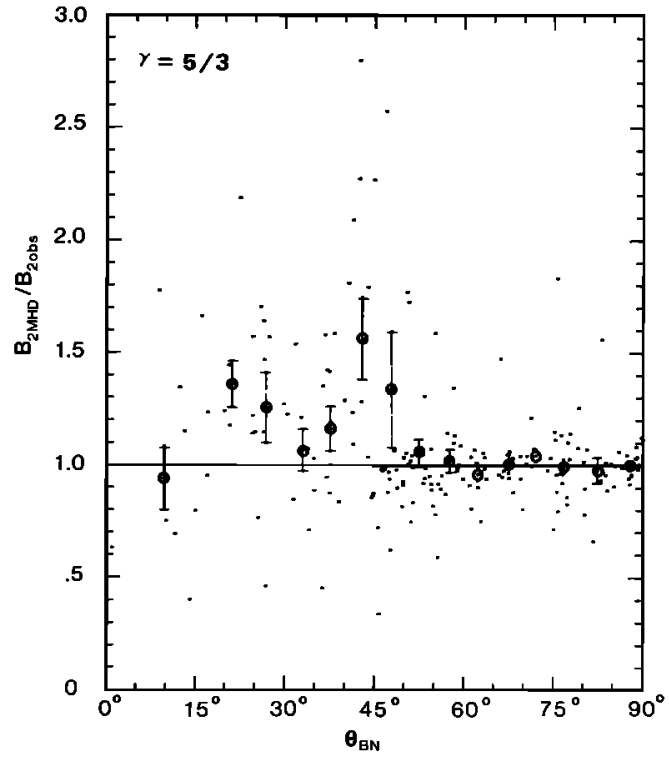

Fig. 8. Comparison of calculated with observed downstream field magnitude as a function of $\theta_{B N}$. The bin sizes are $5^{\circ}$.

independent of beta, i.e., the MHD calculations work reasonably well for all $\beta$. The division of $B_{2 \mathrm{MHD}} / B_{2 \mathrm{obs}}$ versus $\beta$ into regimes with $\theta_{B N}$ greater than $45^{\circ}$ (Figure $10 b$ ) and $\theta_{B N}$ less than $45^{\circ}$ (Figure 10c) shows that any departure from 1 occurs dominantly in the quasi-parallel cases.

We also calculated the downstream field magnitude using the gas dynamic approach [e.g., Spreiter et al., 1966]. Figure 11 shows a plot of $B_{2 \text { gas }} / B_{2 \text { obs }}$ versus the plasma beta. We see that for $\beta \leqslant 2.5$ (75\% of all cases) the gas dynamic calculations overestimate the observations. In solar wind conditions where the particle pressure dominates the magnetic field pres-
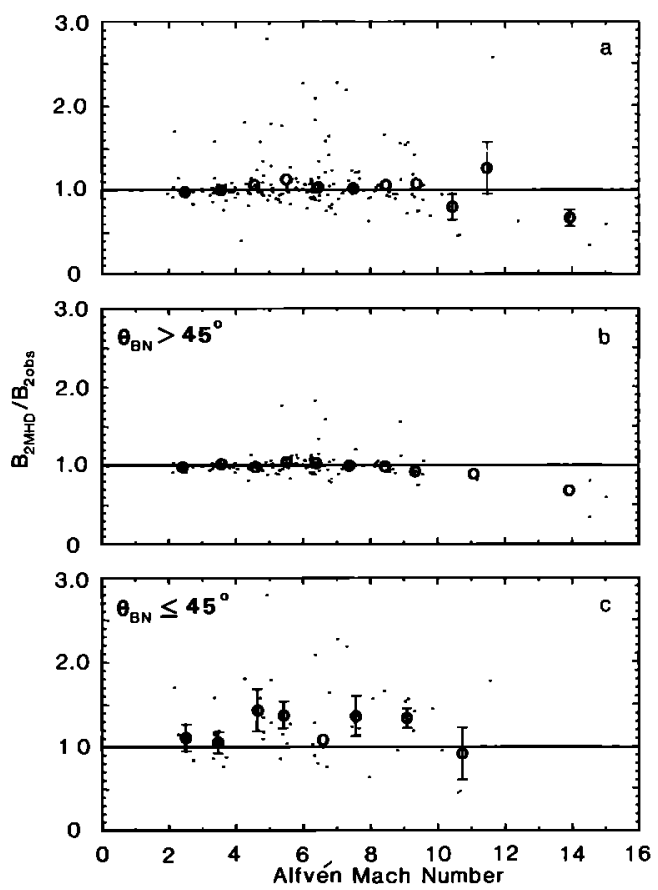

Fig. 9. Comparison of $B_{2 M H D}$ with $B_{2 o b s}$ as a function of Alfvén Mach number, (a) for all cases, (b) for cases that have $\theta_{B N}>45^{\circ}$, and (c) for cases that have $\theta_{B N} \leq 45^{\circ}$. 


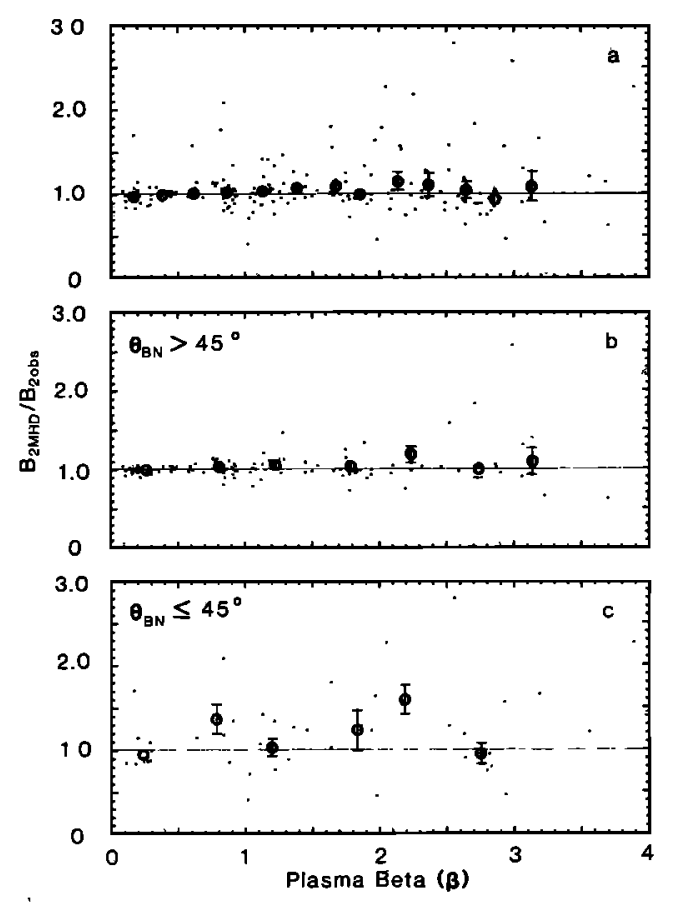

Fig. $1 \theta_{n}$ Comparison of $B_{2 M H D}$ with $B_{20 b s}$ as a function of the plasma betta, (a) for all cases, (b) for cases that have $\theta_{B N}>45^{\circ}$, and (c) for cases that have $\theta_{B N} \leq 45^{\circ}$.

sure $(\beta>2.5)$, the MHD and gas dynamic calculations yield the same results, as expected. A similar result was obtained by Tatrallyay et al. [1984] at the Venus bow shock, where $M a / M s \geq 1.5(\beta \geq 2.7)$ was required for the gas dynamic calculations to agree with MHD calculations, and with observations. ( $M s$ is the sonic Mach number.)

\section{Discussion}

To test the applicability of the simple MHD RankineHugoniot jump condition's to the earth's bow shock, we compared the calculated with the observed jumps in the magnetic field magnitudes. This comparison could have been done as well, of course, with any other parameter such as density or temperature. We chose the field magnitude because almost continuous field measurements are available and because of the relative ease with which a shock can be identified in the high-resolution time series, especially if it is a quasiperpendicular shock.

There is a potential problem, though, in using only magnetic field data to identify quasi-parallel shocks, because their structure is very disordered, and because the shock jump approaches unity as the angle between the interplanetary magnetic field and the shock normal approaches zero. In this situation it is very diflicult to say on the basis of magnetic data alone where the actual transition between the upstream and the downstream states ocurs. In fact, in this statistical study we found it necessary to add a certain fraction (10\%) of quasiparallel shocks whose times had been identified in the plasma data (courtesy of the Los Alamos National Laboratory Plasma Group, in particular J. Gosling) to avoid biasing the data toward the larger and more easily visible magnetic field jumps. With these additional bow shock crossings supplementing a data set obtained from shocks with readily identifiable magnetic signatures for all $\theta_{B N}$, we are assured adequate representation of the quasi-parallel regime.
Quasi-parallel shocks always exhibit a large amount of disorder both upstream and downstream [e.g., Greenstadt, 1974]. Downstream, the variations in the magnetic field are often of the order of the background field itself. In this analysis we compared upstream and downstream field magnitudes with calculations and had to average the magnetic field data. When taking the average of the magnetic field one may do so either by averaging the magnitude $(\langle|\mathbf{B}|\rangle)$ or by averaging the components and then calculating the magnitude $(|\langle B\rangle|)$ [e.g., Sonett et al., 1968]. If we regard the turbulence as fluctuations with zero mean superimposed on all the field components, then averaging the field vector will minimize any contributions from field fluctuations and give a representative value for the background field magnitude. We will make this assumption and thus use $|\langle\dot{\mathbf{B}}\rangle|$ as our averages. In this feature our approach differs from that used by Tatrallyay et al. [1984]

That the choice of averaging procedure is very important is shown in Figures $12 a$ and $12 b$. Here we plot the ratio $|\langle\mathbf{B}\rangle|\left\langle\langle\mathbf{B} \mid\rangle\right.$ versus $\theta_{B N}$ for both upstream and downstream measurements, respectively. We see that for $45^{\circ}<\theta_{B N} \leq 90^{\circ}$ the ratio is unity. However, for $\theta_{B N} \leq 45^{\circ}$, where the fluctuations are large, the difference between the two averaging procedures becomes great, especially for the downstream region. The effect of using $\langle|\mathbf{B}|\rangle$ instead of $|\langle\mathbf{B}\rangle|$ is most evident when $\boldsymbol{B}_{2 \mathrm{MHD}} / \boldsymbol{B}_{2 \mathrm{obs}}$ is plotted versus $\theta_{\boldsymbol{B N}}$. Instead of rising to values greater than 1, however, as in Figure 8 , the field ratio drops with decreasing $\theta_{B N}$, approaching approximately 0.4 in the parallel shock limit. The other features of our comparison, such as the distribution of the polytropic index or the behavior of $B_{2 M H D} / B_{2 o b s}$ versus $M a$ and $\beta$, do not appear to be very sensitive to the choice of the field averaging procedure.

On the question of the polytropic index of the solar wind, we found that the most probable value of $\gamma$ is between 1.60 and 1.70. This range is consistent with a polytropic index of $\frac{5}{3}$ for the solar wind. However, many individual cases are better approximated with other values of $\gamma$. Examining these cases, we find that there is evidence for systematic trends of the ratio of predicted to observed helld magnitudes, as a function of $\theta_{B N}$

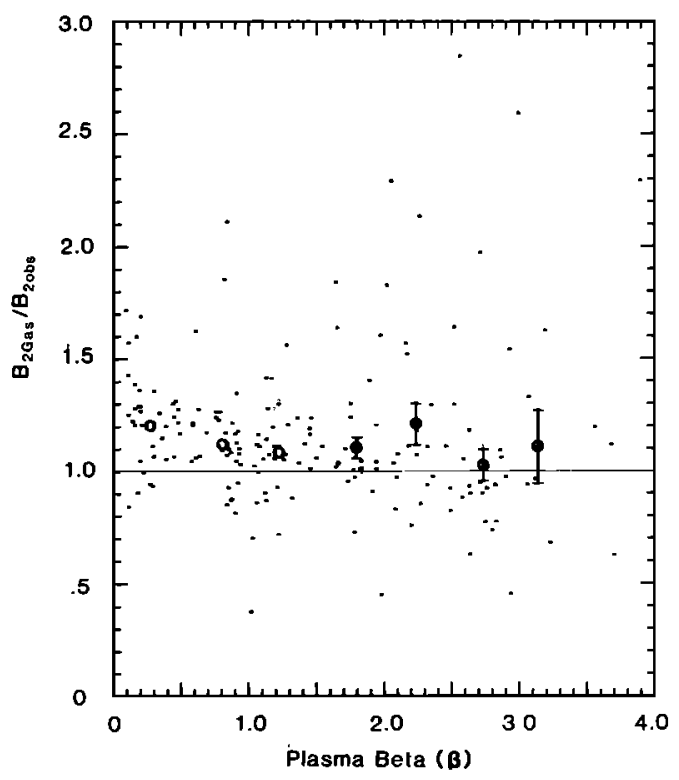

Fig. 11. Comparison of the downstream field magnitude calculated by using the gas dynamic model, with the observed downstream field magnitude as a function of the plasma beta. The bin sizes are 0.5 . 


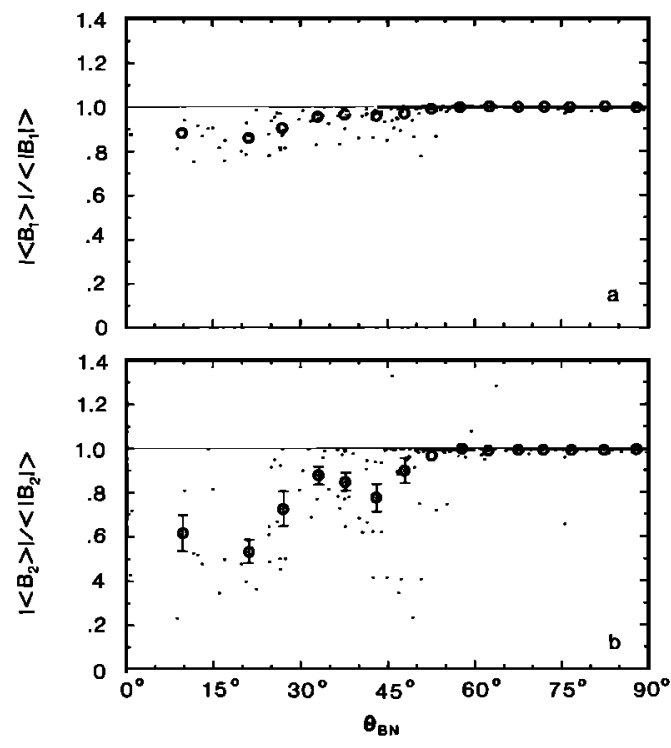

Fig. 12. Comparison of averaging methods, $\langle B\rangle \mid$ and $\langle|B|\rangle$, as a function of $\theta_{B N},(a)$ for upstream and $(b)$ downstream.

and $M a$. In the quasi-perpendicular region $\left(\theta_{B N}>45^{\circ}\right)$ the calculations with $\gamma=\frac{5}{3}$ matched the observations very well, with little scatter present. Below $45^{\circ}$ the predicted downstream field values were generally larger than those observed, but the reason for this disagreement is not clear yet. In the quasiperpendicular shocks examined, the ratio of calculated to observed downstream magnitudes was found to be near 1 , independent of the Alfvén Mach number for $2 \leq M a<10$. Above $M a \cong 10$ there was disagreement, with predictions underestimating the downstream fields, but there were relatively few shocks per bin in this regime.

Without trying to specify any processes at the bow shock that may have caused the disagreements, we find that the spread in the distribution of the apparent polytropic index (Figure 7) was greatest when $\theta_{B N} \leqslant 45^{\circ}$. The distribution of $\gamma$ for cases with $\theta_{B N} \lesssim 45^{\circ}$ is broad (over the range $1 \leq \gamma>3$ ) in comparison with the distribution in Figure 7. Independent of $\theta_{B N}$, most of the high Mach number cases $(M a>10)$ have $\gamma<\frac{5}{3}$.

In thermal equilibrium the polytropic index of a monatomic plasma such as the solar wind is $\frac{5}{3}$, independent of any imposed or self-induced magnetic fields. In our calculations, $\gamma$ is a free parameter, and its departure from $\frac{5}{3}$ may be regarded as evidence that some of our assumptions are not valid. For example, the assumed equilibrium with equal parallel and perpendicular temperatures is not always present. In fact, it is observed [e.g., Feldman et al., 1977] that in the solar wind the ratio of parallel to perpendicular ion temperatures is approximately 1.5 , on the average, so that the assumption of the model calculations may not always be satisfied. Anisotropic pressure modifies plasma behavior and may explain some of the discrepancy between predictions and observations. Other possibilities are that the heat flux (and/or other dissipative processes), neglected in our treatment, may be of importance in some instances, or the omission in (9) of the magnetic fluctuation energy, which can be of the same order of magnitude as the particle internal energy in the downstream quasiparallel region, may be inappropriate. While the peak in the $40^{\circ}-45^{\circ}$ bin in Figure 8 may not be statistically significant because of the large error bars, it brings to mind another possibility. It has been shown [Asbridge et al., 1968; Scarf et al., 1970; Benson et al., 1975; Gosling et al., 1978] that near $\theta_{B N}=45^{\circ}$ a part of the solar wind is reflected, energized, and emitted upstream along the solar wind magnetic field. While the number of these reflected ions is usually small ( 1 to $2 \%$ ), they represent a fairly large energy flux going back upstream. Inclusion of an energy flux term for these ions in the energy equation (9) may diminish the difference between calculations and observations in Figure 8.

It is of interest to note that a difference between calculations and observations as a function of Alfvén Mach number occurs at and above $M a \sim 10$. It is in this region of parameter space, approximately, where the occurrence of a second critical Mach number has been proposed, at which a shock in a collisionless plasma is thought to disappear because of excessive particle reflection. Eselevich et al. [1971], in a laboratory experiment, showed the second critical Mach number to occur at $M a \sim 7$. They noted, however, that this critical Mach number is sensitive to the upstream parameters of the plasma and may vary accordingly. Leroy [1983] states that in simulations of shock processes, as well, an upper critical Mach number is encountered at $M a \sim 12$, above which no steady state shock solution can be obtained from the governing equations.

\section{SUMMARY}

In this study of the magnetic field jump across the earth's bow shock we found that predictions from the MHD conservation equations (7) $(10)$ agreed on average with the observed results provided $\theta_{B N}>45^{\circ}$ and $M a<10$. For $\theta_{B N} \leq 45^{\circ}$ the predictions overestimate the observations considerably. In particular, near $\theta_{B N}=45^{\circ}$ the disagreement is most pronounced. For $M a>10$ the predictions tend to underestimate the observations.

Considerable scatter about the mean was found for most ranges of upstream parameters, so in individual cases the MHD predictions can be quite lar off. Treating the polytropic index as a free parameter, a most probable value of $\gamma$ between 1.60 and 1.70 was obtained, a range consistent with $\gamma=\frac{5}{3}$ for the solar wind. We also found that the larger values of the (apparent) polytropic index are obtained for quasi-parallel shock crosșings, while the smaller values occur during high Alfvén Mach number solar wind conditions.

Acknowledgments. This work was supported in part by the National Science Foundation, Division of Atmospheric Science, under grant ATM 83-00523, and in part by the University of California, Los Angeles. We would like to thank J. G. Luhmann, M. M. Mellott, M. Tatrallyay, J. Scudder, and J. Spreiter for many helpful discussions. Also, the Plasma Group at the Los Alamos National Laboratory was very helpful in providing the necessary plasma data. This is IGPP publication 2549.

The Editor thanks J. D. Mihalov for his assistance in evaluating this paper.

\section{REFERENCES}

Argo, H. V., J. R. Asbridge, S. J. Bame, A. J. Hundhausen, and I. B. Strong, Observations of solar wind plasma changes across the bow shock, J. Geophys. Res., 72, 1989, 1967.

Asbridge, J. R., S. J. Bame, and I. B. Strong, Outward flow of protons from the earth's bow shock, J. Geophys. Res., 73, 5777, 1968.

Bame, S. J., J. R. Asbridge, H. E. Felthauser, J. P. Glore, G. Paschmann, P. Hemmerich, K. Lehmann, and H. Rosenbauer, ISEE-1 and ISEE-2 Fast Plasma Experiment and the ISEE-1 Solar Wind Experiment, IEEE Trans. Geosci. Electron., GE-16(3), 216, 1978.

Benson, J., J. W. Freeman, H. K. Hills, and R. R. Vondrak, Bow shock protons in the lunar environment, Moon, 14, 19, 1975.

Boyd, T. J. M., and J. J. Sanderson, Plasma Dynamics, Barnes \& Noble, New York, 1969. 
Chao, J. K., Evidence for waves and/or turbulence in the vicinity of shock waves in space, Intern. Note LPS-72, Lab. per il Plasma nello Spazio, Rome, 1972.

Chao, J. K., and B. Goldstein, Modification of the Rankine-Hugoniot relations for shock waves in space, J. Geophys. Res., 77, 5455, 1972.

Colburn, D. S., and C. P. Sonett, Discontinuities in the solar wind, Space Sci. Rev., 5, 439, 1966

de Hoffman, F., and E. Teller, Magneto-hydrodynamic shocks, Phys. Rev., 80, 692, 1950.

Eselevich, V. G., A. G. Es"kov, R.Kh. Kurtmullaev, and A. I. Malyutin, Isomagnetic discontinuity in a collisionless shock wave, Sov. Phys. JETP, Engl. Transl., 33, 1120, 1971.

Feldman, W. C., J. R. Asbridge, S. J. Bame, and J. T. Gosling, Plasma and magnetic fields from the sun, in Solar Output and Its Variation, edited by $\mathbf{O}$. R. White, Colorado Associated University Press, Boulder, Colo., 1977.

Formisano, V., P. C. Hedgecock, G. Moreno, F. Palmiotto, and J. K. Chao, Solar wind interaction with the earth's magnetic field, 2, Magnetohydrodynamic bow shock, J. Geophys. Res., 78, 3731 1973.

Gosling, J. T., J. R. Asbridge, S. J. Bame, G. Paschmann, and N Sckopke, Observations of two distinct populations of bow shock ions in the upstream solar wind, Geophys. Res. Lett., 5, 957, 1978.

Greenstadt, E. W., Structure of the terrestrial bow shock, in Solar Wind Three, edited by C. T. Russell, Inst. of Geophys. and Planet. Phys., University of California, Los Angeles, Calif., 1974.

Holzer, R. E., T. G. Northrop, J. V. Olson, and C. T. Russell, Study of waves in the earth's bow shock, J. Geophys. Res., 77, 2264, 1972.

Hundhausen, A. J., Coronal Expansion and Solar Wind, SpringerVerlag, New York, 1972.

Jeffrey, A., and T. Taniuti, Non-Linear Wave Propagation, Academic, Orlando, Fla., 1964.

Kantrowitz, A., and H. E. Petschek, Plasma Physics in Theory and Application, edited by W. Kunkel, McGraw-Hill, New York, 1966.

Leroy, M. M., Structure of perpendicular shocks in collisionless plasma, Phys. Fluids, 26, 2742, 1983.

Mihalov, J. D., C. P. Sonett, and J. H. Wolfe, MHD RankineHugoniot equations applied to earth's bow shock, J. Plasma Phys., $3,449,1969$

Russell, C. T., The ISEE-1 and 2 fluxgate magnetometers, IEEE Trans. Geosci. Electron., GE-16(23), 239, 1978.

Scarf, F. L., R. W. Fredricks, L. A. Frank, C. T. Russell, P. J. Cole- man, and M. Neugebauer, Direct correlation of large-amplitude waves with suprathermal protons in the upstream solar wind, $J$. Geophys. Res., 75, 7316, 1970.

Slavin, J. A., and R. E. Holzer, Solar wind flow about the terrestria planets, 1, Modeling bow shock position and shape, J. Geophys. Res., 86, 11,401, 1981.

Slavin, J. A., R. E. Holzer, J. R. Spreiter, S. S. Stahara, and D. S. Chaussee, Solar wind flow about the terrestrial planets, 2, Comparison with gas dynamic theory and implications for solarplanetary interaction, $J$. Geophys. Res., 88, 19, 1983.

Sonett, C. P., D. S. Colburn, R. G. Currie, and J. D. Mihalov, The geomagnetic tail: Topology, reconnection and interaction with the moon, in Physics of the Magnetosphere, edited by R. L. Carovillano, J. F. McClay, and H. R. Radoshi, D. Reidel, Hingham, Mass., 1968.

Sonnerup, B. U. O., and L. J. Cahill, Jr., Magnetopause structure and altitude from Explorer 12 observations, J. Geophys. Res., 72, 171, 1967.

Spreiter, J. R., A. L. Summers, and A. Y. Alksne, Hydromagnetic flow around the magnetosphere, Planet. Space Sci., 14, 223, 1966.

Spreiter, J. R., A. L. Summers, and A. Y. Alksne, On the comparison of temperature jumps across the earth's shock: VELA 3 satellites and gas dynamic theory, J. Geophys. Res., 73, 1851, 1968.

Tatrallyay, M., C. T. Russell, J. G. Luhmann, A. Barnes, and J. D. Mihalov, On the proper Mach number and ratio of specific heat for modeling the Venus bow shock, J. Geophys. Res., 89, 7381, 1984.

Tidman, D. A., and N. A. Krall, Shock Waves in Collisionless Plasmas, Wiley-Interscience, New York, 1971.

Winterhalter, D., M. G. Kivelson, R. J. Walker, C. T. Russell, and K. B. Quest, High Mach number terrestrial bow shock observations (abstract), Eos Trans. AGU, 65, 1072, 1984.

Zhuang, H. C., and C. T. Russell, An analytic treatment of the structure of the bow shock and magnetosheath, J. Geophys. Res., 86, $2191,1981$.

M. G. Kivelson, C. T. Russell, R. J. Walker, and D. Winterhalter, Institute of Geophysics and Planetary Physics, University of California, Los Angeles, CA 90024.

(Received June 18, 1984;

revised October 19,1984

accepted October 22, 1984.) 